\title{
Acetaminophen administration and the risk of acute kidney injury: a self-controlled case series study
}

This article was published in the following Dove Press journal: Clinical Epidemiology

\author{
Shusuke Hiragi ${ }^{1,2}$ \\ Hiroyuki Yamada' \\ Tatsuo Tsukamoto1,3 \\ Kazuki Yoshida ${ }^{4,5}$ \\ Naoya Kondo' \\ Takeshi Matsubara' \\ Motoko Yanagita' \\ Hiroshi Tamura ${ }^{2}$ \\ Tomohiro Kuroda ${ }^{2}$ \\ 'Department of Nephrology, \\ Graduate School of Medicine, Kyoto \\ University, ${ }^{2}$ Division of Medical \\ Informatics and Administration \\ Planning, Kyoto University Hospital, \\ Kyoto, ${ }^{3}$ Department of Nephrology \\ and Dialysis, Kitano Hospital, \\ Tazuke Kofukai Medical Research \\ Institute, Osaka, Japan; ${ }^{4}$ Department \\ of Epidemiology, ${ }^{5}$ Department of \\ Biostatistics, Harvard T.H. Chan \\ School of Public Health, Boston, \\ MA, USA
}

Correspondence: Shusuke Hiragi Division of Medical Informatics and Administration Planning, Kyoto University Hospital, 54 Kawaharacho, Shogoin, Sakyo-ku, Kyoto 606-8507, Japan

Tel +8I 753667703

$\mathrm{Fax}+81753667704$

Email hiragi.shusuke.4x@kyoto-u.ac.jp
Background: Acetaminophen (APAP) is frequently used for analgesia and is considered safer than nonsteroidal anti-inflammatory drugs (NSAIDs) for the kidneys. However, there is little epidemiological evidence of the association between APAP and acute kidney injury (AKI).

Objectives: To examine the association between APAP and AKI using the self-controlled case series (SCCS) method, which is a novel strategy to control between-person confounders by comparing the risk and reference periods in each patient.

Methods: We performed SCCS in 1,871 patients (39.9\% female) who were administered APAP and subsequently developed AKI, by reviewing electronically stored hospital information system data from May 2011 to July 2016. We used conditional Poisson regression to compare each patient's risk and reference period. As a time-varying confounder, we adjusted the status of liver and kidney functions, systemic inflammation, and exposure to NSAIDs.

Results: We identified 5,650 AKI events during the 260,549 person-day observation period. The unadjusted incidences during the reference and exposure periods were 2.01/100 and 3.12/100 person-days, respectively. The incidence rate ratio adjusted with SCCS was 1.03 ( $95 \%$ confidence interval [CI]: 0.95-1.12). When we restricted endpoints as stage 2 AKI- and stage 3 AKI-level creatinine elevations, the incidence rate ratios were 1.20 (95\% CI $0.91-1.58)$ and $1.20(95 \%$ CI 0.62-2.31), respectively, neither of which was statistically significant.

Conclusion: Our findings added epidemiological information for the relationship between APAP administration and AKI development. The results indicated scarce association between APAP and AKI, presumably supporting the general physicians' impression that APAP is safer for kidney. Keywords: acetaminophen, acute kidney injury, adverse drug event, self-controlled case series, hospital information system

\section{Introduction}

Acetaminophen ( $N$-acetyl-para-amino-phenol [APAP]) is a frequently used pain killer. APAP is generally regarded as a safer drug with regard to kidney function compared to nonsteroidal anti-inflammatory drugs (NSAIDs), which are known to contribute to the development of acute kidney injury (AKI). ${ }^{1}$ However, little is known about the association between APAP and AKI, especially for therapeutic dosing.

Previous case reports ${ }^{2-4}$ and cohort studies ${ }^{5,6}$ have suggested an association between supratherapeutic doses of APAP and AKI. To our knowledge, there is only one case series $^{7}$ that reported two AKI cases suspiciously induced by therapeutic-dose APAP. The causality in those cases remains unclear because the patients had taken other suspicious drugs in addition to APAP, and the drug-induced lymphocyte stimulation tests, which 
examined their hypersensitivity to APAP, were negative. ${ }^{7}$ Looking at in vitro data, APAP in therapeutic doses is said to induce fibroblast proliferation, possibly resulting in kidney injury, but its clinical implications have not yet been proven. ${ }^{8}$ Although there is quite limited epidemiological evidence for the association between therapeutic-dose APAP and AKI, guidelines, ${ }^{9,10}$ textbook, ${ }^{11}$ and even the National Kidney Foundation web page ${ }^{12}$ describes its safer profile for the kidney, which is widely known among physicians. ${ }^{13}$ In contrast, an official medical package leaflet published in Japan ${ }^{14}$ stated that APAP is contraindicated in patients with severe renal impairment in order to prevent further renal damage, but no evidence has been shown to reinforce this contraindication.

Therefore, we believe that it is important to investigate any association between therapeutic dose APAP and AKI, to enhance better decision making for pain management. However, APAP is so widely used ${ }^{13}$ that demographic variance of the target population is too large; many between-person confounding factors would exist if we intended to perform conventional retrospective methods. Hence, a contrivance in study design is required.

In this study, we aimed to demonstrate the possible association between the administration of therapeutic doses of APAP and the occurrence of AKI, using a recently introduced, retrospective, observational design named self-controlled case series (SCCS). As a model, SCCS can easily control for potential between-person confounding factors that do not change over time by comparing risk and reference periods within each individual. ${ }^{15,16}$

\section{Methods}

\section{Study design and settings}

This is an SCCS study using drug prescription and biochemical blood test data obtained from the hospital information system
(HIS) of a single facility. SCCS is a kind of case-series method developed recently. ${ }^{15,16}$ The method compares incident rates during time periods with different risk statuses within each individual in order to estimate the incident rate ratio of risk factors. ${ }^{17}$ SCCS can therefore avoid time-independent confounding factors occurring between study subjects by comparing "risk" and "reference" periods within individuals; thus, there is no need for a separate control group. This feature makes it easier to examine drug adverse events retrospectively using HIS, in which a suitable control group is difficult to find. ${ }^{18}$ The pictorial representation of the design is shown in Figure 1.

We identified people who were prescribed oral APAP from May 2011 to July 2016, by searching the HIS database of Kyoto University Hospital. The hospital is a tertiary general hospital with 34 divisions including internal medicine, surgery, emergency department, and psychiatry that take care of both the acute and chronic conditions of various diseases. The database covers all the patients who have ever consulted doctors working in the hospital. Its prescription database includes all outpatient and inpatient prescriptions in the hospital but does not include those written outside the hospital (eg, by general practitioners). From these potentially eligible patients, we excluded patients younger than 18 years at the beginning of the observation period, because our research scope was adult patients. In addition, we excluded patients on chronic dialysis therapy who would no longer be burdened by the initiation of renal replacement therapy (RRT). Similar to the prescription, our database does not include any treatment outside the hospital, such as at dialysis facilities. Furthermore, because acute blood purification in the hospital is recorded using paper documents, we could not extract the exact day of RRT. Therefore, we excluded patients whose estimated glomerular filtration rate (eGFR) value at the beginning of the observation was $<7 \mathrm{~mL} / \mathrm{min} / 1.73 \mathrm{~m}^{2}$, as

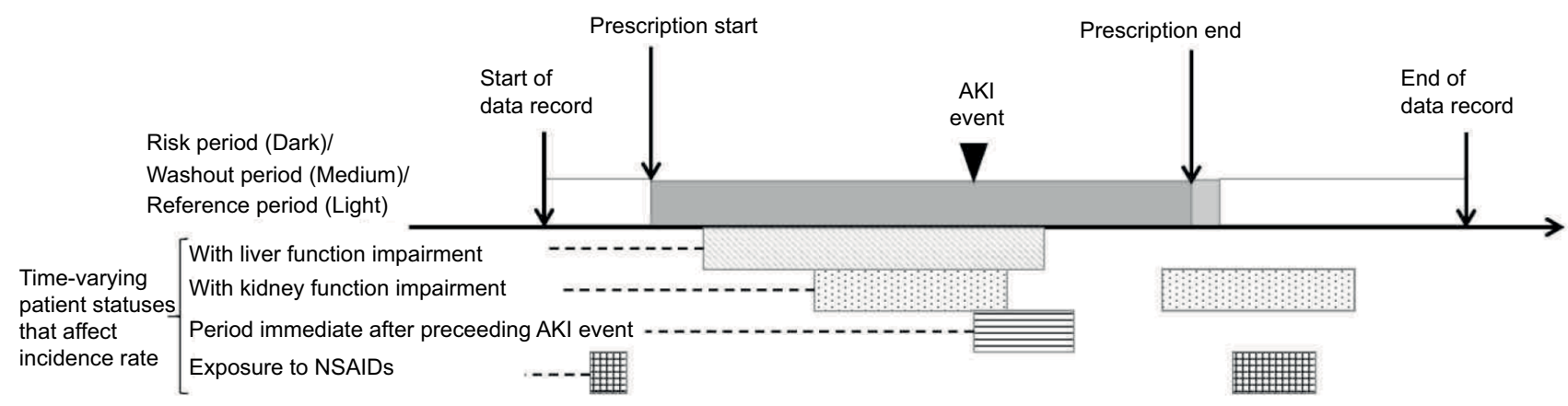

Figure I Pictorial representation of SCCS we used in our context of interest.

Notes: The method compares the incidence rates of risk and reference periods with an adjustment for variables affecting the incidence rate. Here, the risk is acetaminophen administration extracted from prescription data. In this study, we adjusted for exposure to NSAIDs, liver function impairment, kidney function impairment, and effects of preceding AKI event.

Abbreviations: AKI, acute kidney injury; NSAIDs, nonsteroidal anti-inflammatory drugs; SCCS, self-controlled case series. 
calculated with MDRD formula for the Japanese population, because patients with an eGFR $<7 \mathrm{~mL} / \mathrm{min} / 1.73 \mathrm{~m}^{2}$ can be seen as end-stage renal disease (ESRD) patients. ${ }^{19}$

The inclusion flow diagram is shown in Figure 2. This study was approved by the Ethics Committee of Kyoto University Graduate School and Faculty of Medicine (R0290). The Ethical Guidelines for Medical and Health Research Involving Human Subjects in Japan, on which committee approval is based, advised consent from patients was not required because we secondarily used existing patient data collected for clinical purpose. Patient recruitment was done with an adequately managed opt-out method, as required in aforementioned guideline: we disclosed documents showing their right to decline participation to patients. Personal information was handled using secure computers in accordance with the Guideline on the Personal Information Protection Law in Japan.

\section{Exposure and risk period}

We defined our exposure group as those patients who were administered APAP, as identified by data extracted from the HIS of Kyoto University Hospital in the aforementioned period. We used the Japanese drug code specific to oral APAP, which is identical to the Anatomical Therapeutic Chemical (ATC) Classification of D00217, in our database search queries. A risk period in SCCS ("exposed period" in this study) is a period during which the individuals are biologically considered to be at risk of the drug's adverse event of interest. We define the risk period here by the calendar days of APAP administration. If a patient was instructed to take APAP regularly, the corresponding days were considered to be the exposed period, assuming that all patients took all drugs when prescribed. If a patient was prescribed APAP to be taken on an as-needed basis, we assumed that he/she took the medication regularly three times a day and the corresponding periods were seen as the exposed period.

\section{Endpoints and event measures}

The primary endpoint was the occurrence of an AKI event. An AKI event was defined as serum creatinine elevation by $\geq 0.3 \mathrm{mg} / \mathrm{dL}$ in 2 days or $\geq 1.5$ times in 7 days compared to the result of the most recent examination. These criteria were based on the definition of stage 1 AKI by the Kidney Disease Improving Global Outcomes Foundation. ${ }^{20}$ Therefore, in this study, we included only periods in which the patients received blood examinations at least twice in 7 days, based on our method of defining AKI. The onset date of AKI was determined by laboratory data that satisfied the aforementioned criteria. Our blood examination database includes examination date and result but not the exact time

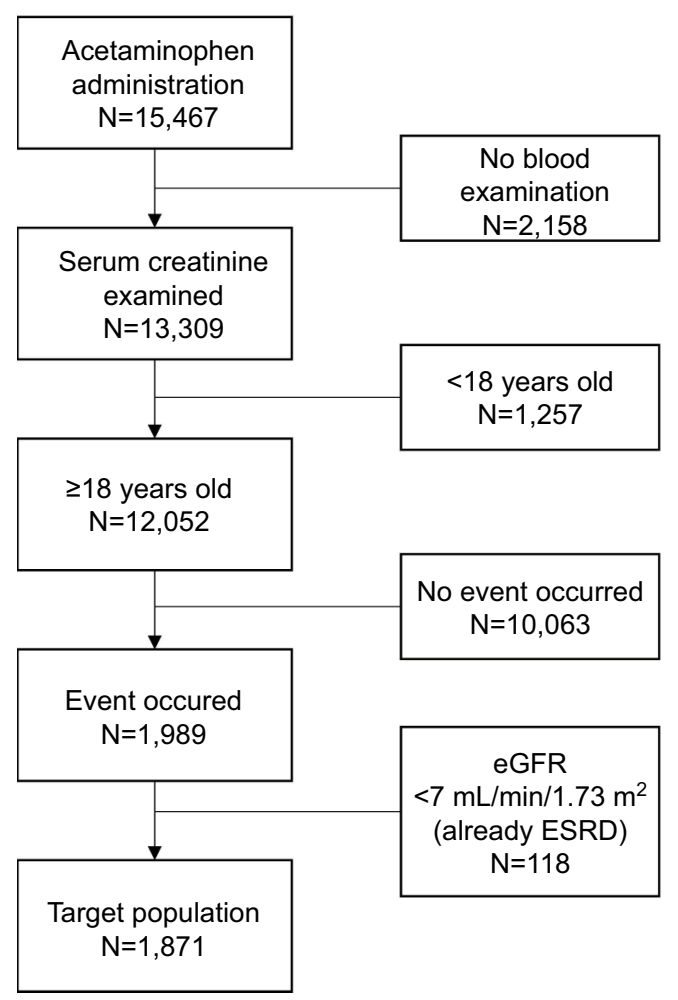

Figure 2 Inclusion flow diagram of the target population. Abbreviations: eGFR, estimated glomerular filtration rate; ESRD, end-stage renal disease.

when the blood specimen was collected. In a precise sense, KDIGO defines stage $1 \mathrm{AKI}$ as a serum creatinine elevation by $\geq 0.3 \mathrm{mg} / \mathrm{dL}$ in $48 \mathrm{~h}$; however, in this study, we used two calendar days instead of $48 \mathrm{~h}$ because of the aforementioned limitation of data granularity.

We also examined the incidence of advanced AKI events as endpoints. Stage 2 AKI (defined as serum creatinine elevation of greater than or equal to twice the baseline level) and stage $3 \mathrm{AKI}$-level creatinine elevation $(\geq 4.0 \mathrm{mg} / \mathrm{dL}$, or greater than or equal to three times the baseline level) were selected. As mentioned earlier, we could not extract the exact date of RRT, hence we applied creatinine elevation condition only as a definition of severer AKI equivalent to stage $3 \mathrm{AKI}$.

\section{Washout period}

Serum creatinine, which is the key value defining AKI, is reported to elevate 24 and $48 \mathrm{~h}$ after kidney damage. ${ }^{21}$ In addition, little is known about the lead time between APAP administration and the onset of kidney injury. Therefore, an AKI event could be detected shortly after the cessation of APAP exposure. In this study, we treated two calendar days after the cessation as a washout period, which had a different risk structure than the control period. We also modified the assumption for the length of the washout period with the sensitivity analysis described later. 


\section{Adjustment of time-varying confounders}

As discussed earlier, SCCS can avoid between-person confounders that do not change over time, but we had to adjust time-varying variables that correlate to both the exposure and the occurrence of the event. Figure 3 shows the causal diagram of our context of interest. We identified several time-varying confounders: liver function impairment, kidney function impairment, systemic inflammation, and NSAIDs use. In addition, we adjusted the effect of AKI occurrence itself on subsequent events.

\section{Liver function impairment}

APAP has been shown to induce renal damage in patients with impaired liver function. ${ }^{5}$ In addition, physicians may hesitate to prescribe APAP for patients with impaired liver function, due to its well-known hepatotoxicity. Therefore, liver function acts as a confounding factor that needs to be adjusted. We defined liver function impairment as the elevation of serum alanine aminotransferase to levels of more than double the upper limits of normal (ULN), or the elevation of alkaline phosphatase above its ULN, referring to the criteria of drug-induced liver injury. ${ }^{22} \mathrm{We}$ assumed liver function impairment to be transient and to occur independently of the kidney injury.

\section{Kidney function impairment}

Kidney function declines gradually over time, ${ }^{23}$ and impaired kidney function is revealed to be the risk factor of AKI development. ${ }^{24,25}$ In addition, patients with impaired kidney function are more likely to be prescribed APAP, ${ }^{26}$ adhering to the recommendation of guideline ${ }^{27}$ and others discussed earlier. ${ }^{9-12}$ Therefore, kidney function is also a time-varying confounder to be adjusted. In this study, we defined kidney function impairment to be an eGFR value of $<60 \mathrm{~mL} /$ $\mathrm{min} / 1.73 \mathrm{~m}^{2}$ and subdivided them into four strata $(45 \leq \mathrm{eGFR}$ $<60 \mathrm{~mL} / \mathrm{min} / 1.73 \mathrm{~m}^{2}, 30 \leq \mathrm{eGFR}<45 \mathrm{~mL} / \mathrm{min} / 1.73 \mathrm{~m}^{2}$, $15 \leq \mathrm{eGFR}<30 \mathrm{~mL} / \mathrm{min} / 1.73 \mathrm{~m}^{2}$, and $7 \leq \mathrm{eGFR}<15 \mathrm{~mL} /$ $\left.\min / 1.73 \mathrm{~m}^{2}\right){ }^{28}$

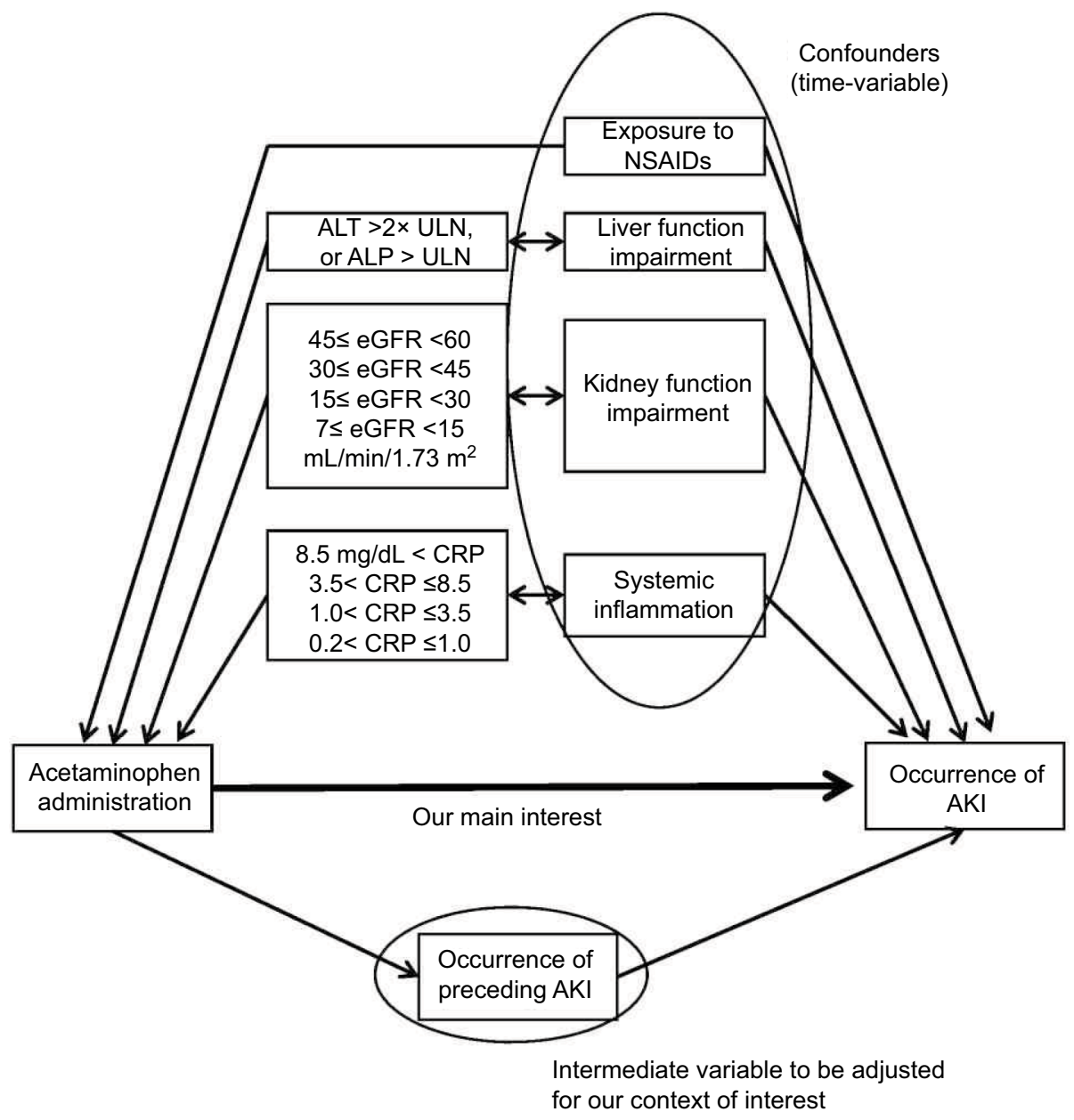

Figure 3 Causal diagram of our context of interest.

Note: We identified and adjusted four time-variable confounders and one intermediate variable shown here.

Abbreviations: AKI, acute kidney injury; ALP, alkaline phosphatase; ALT, alanine aminotransferase; CRP, C-reactive protein; eGFR, estimated glomerular filtration rate; NSAIDs, nonsteroidal anti-inflammatory drugs; ULN, upper limits of normal. 


\section{Systemic inflammation}

AKI occurs in $22.7 \%$ of hospitalized patients, especially those in a severely ill condition. ${ }^{29,30}$ Clinically ill conditions are generally transient and such patients tend to need APAP as analgesic agents, hence, the general condition of the patient is a time-variable confounder. As an indicator of the patients' general condition, we used C-reactive protein (CRP) to represent the status of systemic inflammation. We assumed that a CRP value of $>0.2 \mathrm{mg} / \mathrm{dL}$ indicated systemic inflammation because this value is the threshold in the Kyoto University Hospital laboratory. In addition, we subdivided systemic inflammation status into four strata, decided by the 50th, 75th, and 90th percentiles of the analyzed period sorted by the CRP value $(0.2<\mathrm{CRP} \leq 1.0 \mathrm{mg} / \mathrm{dL}, 1.0<\mathrm{CRP}$ $\leq 3.5 \mathrm{mg} / \mathrm{dL}, 3.5<\mathrm{CRP} \leq 8.5 \mathrm{mg} / \mathrm{dL}$, and $8.5 \mathrm{mg} / \mathrm{dL}<\mathrm{CRP}$ ).

We assumed that aforementioned impaired liver and kidney functions and systemic inflammation status start at the point of the middle day in the blood examination interval. For example, when a patient satisfied impaired liver function criteria at the examination on May 5, while he/she did not satisfy the criteria at the examination just before on May 1, we assumed that his/her impaired liver function started at May 3.

\section{NSAIDs' use}

As discussed earlier, NSAIDs is well known to cause AKI. Both NSAIDs and APAP are analgesics; thus, a correlation in their administration may exist due to background conditions. Therefore, we treated NSAIDs' administration as a confounding factor. We used the Japanese drug code equivalent to the ATC drug code of M01 AB, M01AC, M01AE, M01AG, and M01AH to determine the NSAIDs used. Similar to APAP administration, we defined a 2-day washout period.

\section{Occurrence of AKI}

We intended to examine the direct effect of APAP administration on AKI events, but the occurrence of AKI increases the incidence rate of subsequent AKI. ${ }^{31}$ This means that an AKI event acted as an intermediate variable in our causal model. To the best of our knowledge, there is no firm knowledge about how long the preceding AKI affects the following ones. Therefore, we assumed a 7-day period after AKI (hereafter called the preceding AKI-affected period), to adjust for the effect of one AKI event on subsequent ones, ${ }^{2}$ as shown in Figure 1.

\section{Sensitivity analysis}

To confirm our results, we performed sensitivity analyses. First, we stratified patients by their gender. Next, we modified these assumptions in our analysis: the preceding AKI- affected period (1, 3, and 14 days) and washout period for APAP administration ( 1,7 , and 14 days).

We also calculated the incidence rate ratio of APAP administration with the model including potentially nephrotoxic agents known to cause kidney damage. These drugs could be associated with the development of AKI, but administration of these drugs, except NSAIDs, does not directly correlate to that of APAP because of the difference in their effects. Therefore, they do not necessarily act as confounding factor. The list of drugs is shown in Table S1. ${ }^{32}$

\section{Statistical analysis}

All analyses used R Version 3.2.1 software, ${ }^{33}$ and the results are presented with $95 \%$ confidence intervals (CIs) when appropriate. We used conditional Poisson regression to compare each patient's risk and reference periods with Generalized Nonlinear Models package. ${ }^{34} \mathrm{~A}$ two-sided $P$-value of $<0.05$ was considered statistically significant.

\section{Results}

Patient characteristics in this study are shown in Table 1. We included 1,871 patients, and 39.9\% of the target population was female. The average age at the beginning of the observation was 63.3 years, and the average observation length was 139 days. The average exposure period of APAP administration was 16 days. In our population, $48.6 \%$ of patients had eGFR values $>60 \mathrm{~mL} / \mathrm{min} / 1.73 \mathrm{~m}^{2}$ at the time of first blood examination, while in $32.3 \%$ of patients, the eGFR was between 30 and 60 and, in $19.1 \%$ of patients, the eGFR was between 7 and 29. As explained earlier, patients whose eGFR value was $<7 \mathrm{~mL} / \mathrm{min} / 1.73 \mathrm{~m}^{2}$ were excluded.

Table 2 shows the unadjusted incidence of AKI stratified by the aforementioned factors and incidence rate ratios adjusted with SCCS. There were 5,650 AKI events (33.1\% of them were first time AKI) that occurred during the 260,549 person-days of observation period. Among them, 4,584 AKI

Table I Patient characteristics of included patients $(n=I, 87 I)$

\begin{tabular}{ll}
\hline Characteristics & Value \\
\hline Age, mean ( \pm standard deviation) & 63.3 I $( \pm 15.90)$ \\
Female gender (number of patients, \%) & $747(39.9)$ \\
Baseline eGFR (number of patients, \%) & \\
$\geq 60$ & $909(48.6)$ \\
$30-59$ & $604(32.3)$ \\
$8-29$ & $358(19.1)$ \\
Observation length (days, interquartile range in & $139.3(57-174)$ \\
parenthesis) & \\
Exposure length (days, interquartile range in & $16.06(4-18)$ \\
parenthesis) & \\
\hline
\end{tabular}

Abbreviation: eGFR, estimated glomerular filtration rate. 
Table 2 Unadjusted incidence and adjusted incidence rate ratio of $\mathrm{AKI}$ in periods with each risk status

\begin{tabular}{|c|c|c|c|c|c|}
\hline Risk status & $\begin{array}{l}\text { AKI occurred } \\
\text { (times) }\end{array}$ & $\begin{array}{l}\text { Period length } \\
\text { (person-day) }\end{array}$ & $\begin{array}{l}\text { Incidence (unadjusted/ } \\
\text { I } 00 \text { person-day, } 95 \% \mathrm{CI} \text { ) }\end{array}$ & $\begin{array}{l}\text { Incidence rate ratio } \\
\text { (adjusted with SCCS, } \\
95 \% \mathrm{CI} \text { ) }\end{array}$ & $P$ \\
\hline \multicolumn{6}{|l|}{ Exposure to acetaminophen } \\
\hline No acetaminophen administration & 4,584 & 228,036 & $2.01(1.95-2.07)$ & Reference & \\
\hline With acetaminophen administration & 939 & 30,053 & $3.12(2.93-3.33)$ & $1.03(0.95-1.12)$ & 0.49 \\
\hline Washout periods ( 2 days) & 127 & 2,460 & $5.16(4.32-6.11)$ & $1.05(0.87-1.27)$ & 0.59 \\
\hline \multicolumn{6}{|l|}{ Time-varying confounders } \\
\hline \multicolumn{6}{|l|}{ NSAIDs use } \\
\hline No NSAIDs administration & 4,877 & 229,009 & $2.13(2.07-2.19)$ & Reference & \\
\hline With NSAIDs administration & 681 & 30,096 & $2.26(2.10-2.44)$ & $1.27(1.14-1.4 I)$ & $<0.01$ \\
\hline NSAIDs washout period ( 2 days) & 92 & $\mathrm{I}, 444$ & $6.37(5.17-7.76)$ & $1.24(0.99-1.55)$ & 0.07 \\
\hline \multicolumn{6}{|l|}{ Status of systemic inflammation } \\
\hline $\mathrm{CRP} \leq 0.2 \mathrm{mg} / \mathrm{dL}$ & 401 & 72,712 & $0.55(0.50-0.61)$ & Reference & \\
\hline $0.2<\mathrm{CRP} \leq 1.0 \mathrm{mg} / \mathrm{dL}$ & 568 & 60,822 & $0.93(0.86-1.01)$ & $1.87(1.62-2.15)$ & $<0.01$ \\
\hline $1.0<\mathrm{CRP} \leq 3.5 \mathrm{mg} / \mathrm{dL}$ & 1,224 & 60,897 & $2.01(1.90-2.12)$ & $3.63(3.17-4.15)$ & $<0.01$ \\
\hline $3.5<\mathrm{CRP} \leq 8.5 \mathrm{mg} / \mathrm{dL}$ & 1,469 & 39,907 & $3.68(3.50-3.87)$ & $6.25(5.45-7.16)$ & $<0.01$ \\
\hline $8.5 \mathrm{mg} / \mathrm{dL}<\mathrm{CRP}$ & 1,988 & 26,211 & $7.58(7.27-7.91)$ & $11.7(10.2-13.5)$ & $<0.01$ \\
\hline \multicolumn{6}{|l|}{ Renal function } \\
\hline Without renal function impairment (eGFR $\geq 60$ ) & 402 & 116,739 & $0.34(0.3 \mathrm{I}-0.38)$ & Reference & \\
\hline $45 \leq \mathrm{eGFR}<60 \mathrm{~mL} / \mathrm{min} / 1.73 \mathrm{~m}^{2}$ & 351 & 43,311 & $0.81(0.73-0.90)$ & $7.10(5.91-8.53)$ & $<0.01$ \\
\hline $30 \leq \mathrm{eGFR}<45 \mathrm{~mL} / \mathrm{min} / 1.73 \mathrm{~m}^{2}$ & 874 & 39,889 & $2.19(2.05-2.34)$ & $32.2(27.0-38.5)$ & $<0.01$ \\
\hline $15 \leq \mathrm{eGFR}<30 \mathrm{~mL} / \mathrm{min} / 1.73 \mathrm{~m}^{2}$ & $\mathrm{I}, 754$ & 31,522 & $5.56(5.31-5.82)$ & $143(\mid 19-173)$ & $<0.01$ \\
\hline $7 \leq \mathrm{eGFR}<15 \mathrm{~mL} / \mathrm{min} / 1.73 \mathrm{~m}^{2}$ & 2,269 & 29,088 & $7.80(7.49-8.11)$ & $309(252-379)$ & $<0.01$ \\
\hline \multicolumn{6}{|l|}{ Liver function } \\
\hline Without liver function impairment & 3,006 & 165,586 & $1.82(1.75-1.88)$ & Reference & \\
\hline With liver function impairment & 2,644 & 94,963 & $2.78(2.68-2.89)$ & $1.13(1.05-1.22)$ & $<0.01$ \\
\hline \multicolumn{6}{|l|}{ Time elapsed from the latest $A K I$ event } \\
\hline$\geq 8$ days & 3,330 & 235,748 & I.4I (1.37-I.46) & Reference & \\
\hline$\leq 7$ days & 2,320 & 24,801 & $9.35(8.99-9.72)$ & $1.01(0.95-1.08)$ & 0.68 \\
\hline
\end{tabular}

Abbreviations: AKI, acute kidney injury; Cl, confidence interval; CRP, C-reactive protein; eGFR, estimated glomerular filtration rate; NSAIDs, nonsteroidal antiinflammatory drugs; SCCS, self-controlled case series.

events occurred during 228,036 person-days of the unexposed to APAP period, while 939 events occurred during 30,053 person-days of the exposed period and 127 events did during 2,460 person-days of washout period. The adjusted incidence rate ratio of AKI under the condition of APAP administration was 1.03 (95\% CI: 0.95-1.12), and that of the washout period was 1.05 (95\% CI: 0.87-1.27). Among time-varying confounders, the incidence rate ratios of systemic inflammation, kidney function impairment, liver function impairment periods, and use of NSAIDs were statistically $>1.0$. In particular, during the exposure to NSAIDs, the incidence rate ratio was 1.27 (95\% CI: 1.14-1.41). However, that of the periods immediately after the preceding AKI event did not show statistical significance.

Table 3 shows the incidence rate ratios under the condition of defining endpoints as stage 2 AKI- and stage 3 AKIlevel creatinine elevations. When we define stage $2 \mathrm{AKI}$ as the endpoint, the number of AKI events was 545 and the number of patients that satisfied the inclusion criteria was 399. The incidence rate ratio of APAP administration was
1.20 (95\% CI: 0.91-1.58). When we define stage 3 AKIlevel creatinine elevation as the endpoint, 108 AKI events occurred in 91 patients. The incidence rate ratio was 1.20 (95\% CI: 0.62-2.31).

Finally, we performed sensitivity analyses, as shown in Table 4. When we stratified patients by gender, the incidence rate ratio of APAP administration was 1.03 (men) and 1.01 (women). When we modified the assumptions as to the preceding AKI-affected period and length of the washout period, the incidence rates were $\sim 1.01-1.03$ under all assumptions. When we added potentially nephrotoxic agents into the model, the estimated incidence rate ratio was 1.04 .

\section{Discussion}

We conducted a SCCS study to examine the potential association between APAP administration at therapeutic doses and the occurrence of AKI. Our results showed that the incidence rate of $\mathrm{AKI}$, in the period that patients were prescribed APAP, was slightly higher than in the period in which they were not prescribed APAP, with the conditions of controlling 
Table 3 Incidence rate ratio of AKI under the condition of defining events - stage 2 and stage 3 AKI-level creatinine elevation

\begin{tabular}{|c|c|c|c|c|}
\hline End point definition & AKI stage 2 & $\mathbf{P}$ & AKI stage 3 & $P$ \\
\hline Number of patients & 399 & & 91 & \\
\hline AKI occurred (times) & 545 & & 108 & \\
\hline $\begin{array}{l}\text { Incidence rate ratio of } \mathrm{AKI} \text { in acetaminophen administration period }(95 \% \mathrm{Cl} \\
\text { in parenthesis) }\end{array}$ & $1.20(0.9 \mid-1.58)$ & 0.19 & $1.20(0.62-2.31)$ & 0.59 \\
\hline Incidence rate ratio of $\mathrm{AKI}$ in Washout period ( $95 \% \mathrm{Cl}$ in parenthesis) & $1.26(0.66-2.39)$ & 0.48 & $0.76(0.10-5.87)$ & 0.79 \\
\hline
\end{tabular}

Abbreviations: $\mathrm{AKI}$, acute kidney injury; $\mathrm{Cl}$, confidence interval.

Table 4 Incidence rate ratio of AKI with modified assumptions: result of sensitivity analysis

\begin{tabular}{|c|c|c|c|c|}
\hline Analysis & $\begin{array}{l}\text { Incidence rate ratio of } A K I \\
\text { in acetaminophen administration } \\
\text { period }(95 \% \mathrm{CI})\end{array}$ & $P$ & $\begin{array}{l}\text { Incidence rate ratio of AKI } \\
\text { in washout period }(95 \% \mathrm{Cl})\end{array}$ & $P$ \\
\hline Main analysis & $1.03(0.95-1.12)$ & 0.49 & $1.05(0.87-I .27)$ & 0.59 \\
\hline Only male ( 1,124 patients) & $1.03(0.93-1.15)$ & 0.53 & $1.04(0.82-1.33)$ & 0.73 \\
\hline Only female (747 patients) & $1.01(0.88-1.16)$ & 0.92 & $1.05(0.78-1.42)$ & 0.73 \\
\hline \multicolumn{5}{|l|}{ Preceding AKI-affected period } \\
\hline I day & $1.03(0.95-1.12)$ & 0.49 & $1.05(0.87-1.27)$ & 0.59 \\
\hline 3 days & $1.03(0.95-1.12)$ & 0.52 & $1.05(0.87-1.26)$ & 0.64 \\
\hline 14 days & $1.03(0.95-1.12)$ & 0.47 & $1.07(0.88-1.29)$ & 0.50 \\
\hline \multicolumn{5}{|l|}{ Length of washout period } \\
\hline I day & $1.03(0.95-1.12)$ & 0.47 & $1.15(0.91-1.47)$ & 0.25 \\
\hline 7 days & $\mathrm{I} .02(0.94-I . I I)$ & 0.38 & $0.94(0.83-1.07)$ & 0.38 \\
\hline 14 days & $1.01(0.93-1.10)$ & 0.76 & $0.89(0.80-1.00)$ & 0.05 \\
\hline $\begin{array}{l}\text { Including potentially nephrotoxic agents into the } \\
\text { model }\end{array}$ & $1.04(0.96-1.13)$ & 0.36 & $1.06(0.88-1.28)$ & 0.55 \\
\hline
\end{tabular}

Abbreviations: $\mathrm{AKI}$, acute kidney injury; $\mathrm{Cl}$, confidence interval.

for kidney function, liver function, severity of systemic inflammation, and exposure to NSAIDs as time-varying confounders. However, the difference was not statistically significant over the null hypothesis that APAP administration was not associated with the development of AKI.

The analgesic action of APAP is not fully understood, while its hepatotoxicity is well acknowledged..$^{35}$ Several researchers have indicated that cyclooxygenase- 3 might be a target enzyme of APAP, ${ }^{36,37}$ while other in vivo data have suggested that another metabolite, named AM404, was the key factor in the mechanism of APAP as an analgesic agent. ${ }^{38}$

There are several case reports reporting that an APAP overdose causes AKI. ${ }^{2-4}$ Previous reviews have suggested that renal insufficiency occurs in 1-2\% of patients after an APAP overdose ${ }^{39}$ Another cohort study from Taiwan showed that the overall risk of developing AKI was about twice as high in patients with APAP intoxication, ${ }^{6}$ discussing that AKI may be caused by $N$-acetyl- $p$-benzoquinoneimine (NAPQI), which is a toxic intermediate metabolite of $\mathrm{APAP}^{40}$ produced in excess by the saturation of normal metabolic pathways in the liver. Hence, their results are difficult to apply to the effects of APAP at therapeutic doses.

So far, there are mixed opinions about the association between therapeutic dose APAP and AKI. To our knowledge, there is no observational study examining the association between them. In the present study, we utilized SCCS with the adjustment of time-varying confounders to extract reliable associations from an easily available HIS data, which contains prescription and blood examination results. APAP is a relatively inexpensive drug to compensate costly clinical research; therefore, we believed that it was important to pursue less costly but statistically validated methods.

SCCS was originally developed for evaluating association between vaccinations and their adverse effects ${ }^{15}$ and is now commonly used for the purpose. ${ }^{41}$ Recently, researchers have investigated the associations between drug administration and some events by this method using existing electronically stored databases, with examples including antipsychotic drugs and myocardial infarction ${ }^{42}$ and thiazolidinediones and fracture. ${ }^{43}$ One study investigated the association between antidepressants and hip fracture using both SCCS and conventional case-control method and concluded that SCCS could perform more accurately than case-control methods, ${ }^{44}$ indicating that SCCS is potentially valuable for our research purposes.

Our main analysis secondarily showed that the incidence rates of AKI associated with systemic inflammation, kidney function, liver function, and NSAIDs' use were 
significantly higher than reference. These results were compatible with previous reports, indicating that the analysis program for our SCCS model was properly developed. The effect of preceding AKI events did not show statistical significance in spite of existing knowledge, but this result can be explained by the variable's correlation to kidney function impairment.

Our data showed that the incidence rate of AKI associated with APAP administration was 1.03 times higher than the reference and was 1.05 times higher in the washout period. Point estimates were slightly $>1.0$, but it did not show statistical significance. Not showing statistical significance does not directly mean the absence of a relationship between APAP and AKI, but considering the distribution of the $95 \% \mathrm{CI}$, it could be supposed to be scarce or ignorable. In contrast to the aforementioned case report ${ }^{7}$ and the in vitro data ${ }^{8}$ indicating that therapeutic dose APAP could cause AKI, our result was consistent with the well-known physicians' impression. At least, considering a previous report, ${ }^{1}$ which showed that the AKI risk with NSAIDs' use is $60 \%$ higher than reference, and our present data showing the association of NSAIDs' use with AKI, we could conclude that the clinical importance of the association between APAP use and AKI is weaker than that of NSAIDs.

When we set the event as advanced AKI events, the point estimates of the incidence rate ratios were higher than that of the base case model, but they also did not show statistical significance. Though the number of patients was small and CI became wider, the difference in the incidence rate of severer AKI, part of which can be seen as severe kidney injury as requiring RRT, associated with APAP administration, could be supposed to have little clinical importance.

From the sensitivity analyses, which modified assumptions in the base case model, we obtained almost similar incidence rate ratios, indicating the robustness of our findings. Including potentially nephrotoxic agents into our model also did not show apparent change in the results. This result may be explained by the little correlation between administration of APAP and these drugs. However, it must be considered that we included all drugs grouped together on account of the difficulty in data handling when including each drug separately into the model.

AKI can sometimes be a lethal condition, ${ }^{45}$ and it is also well known that those who recovered from AKI can progress to chronic kidney disease, ${ }^{46}$ which is a major risk factor for cardiovascular diseases. AKI can lower patients' quality of life, ${ }^{47,48}$ and therefore, prevention of AKI is very important. From our results, the clinical importance of the association between APAP and AKI seems unremarkable, or at least smaller than that of NSAIDs and AKI. Considering the side effects of other pain-killing treatment options, using APAP is a reasonable choice when physicians intend to preserve patients' kidney function.

\section{Limitations}

There were several limitations to our study. First, unobserved confounding factors may still exist, including both those arising from the limitations of the dataset and those having intrinsically uncontrollable features. For example, we could not include the major time-varying conditions, such as postcardiac surgery state and sepsis, due to the limitation of our dataset, which could not sufficiently acquire the existence of such conditions. In addition, the indication for APAP administration may act as an intrinsically uncontrollable time-varying confounding factor. We cannot distinguish AKI caused by APAP from AKI due to a background poor clinical condition that resulted in APAP administration. As described in the "Methods" section, those time-varying factors that correlate to APAP administration and affect the risk of AKI development can result in the observed incidence rate ratios being larger or smaller than the real value. From the clinical viewpoint, such confounders generally increase the proportion of APAP administration and also increase the incidence of AKI because severe conditions require pain control and result in high incidence of AKI. Therefore, a calculated incidence rate ratio with only observed confounders, in which APAP administration period contains more patients with unobserved confounder increasing incidence rate, shows larger values than real incidence rate ratios. Considering that our main result already showed little association between APAP and AKI, this problem may have little impact on our result, even though the possibility of an unexpected effect still remained.

Second, we used a dataset from a single facility and, therefore, potential sampling bias may exist. Third, this study restricted observation periods to those periods in which patients received blood examinations at least once in 7 days. That is to say, our results cannot directly be applied to patients who do not need such frequent blood examinations. Last, we could not fully collect APAP administration (eg, taking over-the-counter drugs). This bias can lead to erroneous estimation of the incidence rate ratio, but its effect may be small. This is because Japanese health insurance covers APAP prescription for almost all diseases, so the target population could get an APAP prescription at lower cost than buying over-the-counter drugs. 


\section{Conclusion}

Using prescription and biochemical blood test data from HIS, we performed SCCS to compare the incidence rate of AKI between periods in which patients were and were not exposed to therapeutic dose APAP. We found that the incidence was slightly higher during a period with APAP administration as a point estimate; however, the difference was not statistically significant. General physicians often recognize that APAP is relatively safe for the kidneys; however, it has not been demonstrated in epidemiological data. Even though it is not necessarily a statistically precise interpretation, our research epidemiologically added supporting information to the general physicians' impressions.

\section{Data sharing}

It is difficult for authors to share data we used, because our data contain personal identifier, prescription data, and blood examination data, which Japanese legal regulation regards as sensitive personal information not to be freely disclosed.

\section{Acknowledgments}

We would like to thank Dr Hideki Yokoi, for the valuable advice with regard to nephrology affairs. We gratefully acknowledge Ms Kaori Shiomi, Yoko Hara, and Yuko Furusawa for their secretarial assistance. We also would like to thank Editage (www.editage.jp) for English language editing. This work was supported by the Japan Society for the Promotion of Science (JSPS) KAKENHI grant number JP5549881 and Ministry of Health, Labor and Welfare Research grant number H29-Seisaku-Shitei-005.

\section{Author contributions}

SH and TT contributed to the research idea and study design. SH contributed to the data acquisition. SH and HY analyzed the data. KY and NK contributed to the statistical analysis. SH and HY contributed to the article writing. TM and HT contributed to the mentorship. MY and TK contributed to the supervision. All authors contributed toward data analysis, drafting and revising the paper and agree to be accountable for all aspects of the work.

\section{Disclosure}

The authors report no conflicts of interest in this work.

\section{References}

1. Griffin MR, Yared A, Ray WA. Nonsteroidal antiinflammatory drugs and acute renal failure in elderly persons. Am J Epidemiol. 2000;151(5):488-496.
2. Mazer M, Perrone J. Acetaminophen-induced nephrotoxicity: pathophysiology, clinical manifestations, and management. J Med Toxicol. 2008;4(1):2-6.

3. Loh C, Ponampalam R. Nephrotoxicity associated with acute paracetamol overdose: a case report and review of the literature. Hong Kong J. 2006;13(2):105-110.

4. Blakely P, McDonald BR. Acute renal failure due to acetaminophen ingestion: a case report and review of the literature. J Am Soc Nephrol. 1995;6(1):48-53.

5. Stollings JL, Wheeler AP, Rice TW. Incidence and characterization of acute kidney injury after acetaminophen overdose. J Crit Care. 2016;35:191-194.

6. Chen YG, Lin CL, Dai MS, et al. Risk of acute kidney injury and long-term outcome in patients with acetaminophen intoxication: a nationwide population-based retrospective cohort study. Medicine. 2015;94(46):e2040.

7. Kato H, Fujigaki Y, Inoue R, et al. Therapeutic dose of acetaminophen as a possible risk factor for acute kidney injury: learning from two healthy young adult cases. Int Med. 2014;53(14):1531-1534.

8. Yu YL, Yiang GT, Chou PL, et al. Dual role of acetaminophen in promoting hepatoma cell apoptosis and kidney fibroblast proliferation. $\mathrm{Mol}$ Med Rep. 2014;9(6):2077-2084.

9. Chou R, Qaseem A, Snow V, et al. Diagnosis and treatment of low back pain: a joint clinical practice guideline from the American College of Physicians and the American Pain Society. Ann Intern Med. 2007;147(7):478-491.

10. Pham PC, Khaing K, Sievers TM, et al. 2017 update on pain management in patients with chronic kidney disease. Clin Kidney J. 2017;10(5):688-697.

11. Davison SN. In: Curhan GC, editor. Management of Chronic Pain in Chronic Kidney Disease. Waltham, MA: UpToDate; 2017. Available from: https://www.uptodate.com/contents/management-of-chronicpain-in-chronic-kidney-disease. Accessed January 2, 2018.

12. NKF [webpage on the Internet]. Pain Medicines (Analgesics); 2017. Available from: https://www.kidney.org/atoz/content/painmeds_analgesics. Accessed December 23, 2017.

13. Kaplowitz N. Acetaminophen hepatoxicity: what do we know, what don't we know, and what do we do next? Hepatology. 2004;40(1):23-26.

14. Medical Package Leaflet. Acetaminophen Tab. 200mg "TEVA"; 2016. Available from: http://www.info.pmda.go.jp/downfiles/ph/ PDF/400813_1141007F1179_1_03.pdf. Accessed July 28, 2017.

15. Farrington CP. Relative incidence estimation from case series for vaccine safety evaluation. Biometrics. 1995;51(1):228-235.

16. Whitaker HJ, Farrington CP, Spiessens B, Musonda P. Tutorial in biostatistics: the self-controlled case series method. Stat Med. 2006;25(10): 1768-1797.

17. Langan SM, Minassian C, Smeeth L, Thomas SL. Risk of stroke following herpes zoster: a self-controlled case-series study. Clin Infect Dis. 2014;58(11):1497-1503.

18. Petersen I, Douglas I, Whitaker H. Self controlled case series methods: an alternative to standard epidemiological study designs. $B M J$. 2016;354:i4515.

19. Cooper BA, Branley P, Bulfone L, et al. A randomized, controlled trial of early versus late initiation of dialysis. NEngl JMed. 2010;363(7):609-619.

20. Kidney Disease: Improving Global Outcomes (KDIGO) Acute Kidney Injury Work Group. KDIGO Clinical Practice Guideline for Acute Kidney Injury. Summary of recommendation statements. Kidney Int Suppl. 2012; 2(1):8-12.

21. McIlroy DR, Wagener G, Lee HT. Biomarkers of acute kidney injury: an evolving domain. Anesthesiology. 2010;112(4):998-1004.

22. Danan G, Benichou C. Causality assessment of adverse reactions to drugs - I. A novel method based on the conclusions of international consensus meetings: application to drug-induced liver injuries. J Clin Epidemiol. 1993;46(11):1323-1330.

23. Mitch WE, Walser M, Buffington GA, Lemann J Jr. A simple method of estimating progression of chronic renal failure. Lancet. 1976;2(7999):1326-1328. 
24. Chawla LS, Eggers PW, Star RA, Kimmel PL. Acute kidney injury and chronic kidney disease as interconnected syndromes. $N$ Engl J Med. 2014;371(1):58-66.

25. Moo Park K. Experimental evidence that preexisting chronic kidney disease is a risk factor for acute kidney injury. Kidney Res Clin Prac. 2014;33(2):71-72.

26. Kelkar M, Cleves MA, Foster HR, Hogan WR, James LP, Martin BC. Acute and chronic acetaminophen use and renal disease: a case-control study using pharmacy and medical claims. J Manag Care Pharm. 2012;18(3): 234-246.

27. Henrich WL, Agodoa LE, Barrett B, et al. Analgesics and the kidney: summary and recommendations to the Scientific Advisory Board of the National Kidney Foundation from an Ad Hoc committee of the National Kidney Foundation. Am J Kidney Dis. 1996;27(1):162-165.

28. [No authors listed] Chapter 1: definition and classification of CKD Kidney Int Suppl. 2013;3(1):19-62.

29. Wang HE, Muntner P, Chertow GM, Warnock DG. Acute kidney injury and mortality in hospitalized patients. Am J Nephrol. 2012;35(4):349-355.

30. Cartin-Ceba R, Kashiouris M, Plataki M, Kor DJ, Gajic O, Casey ET. Risk factors for development of acute kidney injury in critically ill patients: a systematic review and meta-analysis of observational studies. Crit Care Res Prac. 2012;2012:691013.

31. Pazhayattil GS, Shirali AC. Drug-induced impairment of renal function. Int J Nephrol Renovasc Dis. 2014;7:457-468.

32. Naughton CA. Drug-induced nephrotoxicity. Am Fam Physician. 2008;78(6):743-750.

33. R Foundation for Statistical Computing [homepage on the Internet]. A language and environment for statistical computing. R Foundation for Statistical Computing; 2015. Available from: https://www.R-project. org/. Accessed January 3, 2018.

34. Turner H, Firth D. Generalized Nonlinear Models in R: An Overview of the gnm Package. 2015. Available from: http://CRAN.R-project.org/ package $=$ gnm. Accessed January 3, 2018.

35. Hinson JA, Roberts DW, James LP. Mechanisms of acetaminopheninduced liver necrosis. Handb Exp Pharmacol. 2010;(196):369-405.

36. Botting RM. Mechanism of action of acetaminophen: is there a cyclooxygenase 3? Clin Infect Dis. 2000;31(supp1 5):S202-S210.
37. Botting R, Ayoub SS. COX-3 and the mechanism of action of paracetamol/acetaminophen. Prostaglandins Leukot Essent Fatty Acids. 2005;72(2):85-87.

38. Hogestatt ED, Jonsson BA, Ermund A, et al. Conversion of acetaminophen to the bioactive $\mathrm{N}$-acylphenolamine AM404 via fatty acid amide hydrolase-dependent arachidonic acid conjugation in the nervous system. J Biol Chem. 2005;280(36):31405-31412.

39. Prescott LF. Paracetamol overdosage. Pharmacological considerations and clinical management. Drugs. 1983;25(3):290-314.

40. da Rocha BA, Ritter AM, Ames FQ, et al. Acetaminophen-induced hepatotoxicity: preventive effect of trans anethole. Biomed Pharm. 2017;86:213-220

41. Weldeselassie YG, Whitaker HJ, Farrington CP. Use of the self-controlled case-series method in vaccine safety studies: review and recommendations for best practice. Epidemiol Infect. 2011;139(12):1805-1817.

42. Brauer R, Smeeth L, Anaya-Izquierdo K, et al. Antipsychotic drugs and risks of myocardial infarction: a self-controlled case series study. Eur Heart J. 2015;36(16):984-992.

43. Douglas IJ, Evans SJ, Pocock S, Smeeth L. The risk of fractures associated with thiazolidinediones: a self-controlled case-series study. PLoS Med. 2009;6(9):e1000154.

44. Hubbard R, Farrington P, Smith C, Smeeth L, Tattersfield A. Exposure to tricyclic and selective serotonin reuptake inhibitor antidepressants and the risk of hip fracture. Am J Epidemiol. 2003;158(1):77-84.

45. Wald R, Quinn RR, Luo J, et al. Chronic dialysis and death among survivors of acute kidney injury requiring dialysis. JAMA. 2009;302(11): 1179-1185.

46. Ponte B, Felipe C, Muriel A, Tenorio MT, Liano F. Long-term functional evolution after an acute kidney injury: a 10-year study. Nephrol Dial Transplant. 2008;23(12):3859-3866.

47. James MT, Ghali WA, Tonelli M, et al. Acute kidney injury following coronary angiography is associated with a long-term decline in kidney function. Kidney Int. 2010;78(8):803-809.

48. Perlman RL, Finkelstein FO, Liu L, et al. Quality of life in chronic kidney disease (CKD): a cross-sectional analysis in the Renal Research Institute-CKD study. Am J Kidney Dis. 2005;45(4):658-666. 


\section{Supplementary material}

Table SI The list of potentially nephrotoxic agents we used in sensitivity analysis

\begin{tabular}{|c|c|}
\hline Potentially nephrotoxic agents & ATC code \\
\hline \multicolumn{2}{|l|}{ Antidepressants/mood stabilizers } \\
\hline Amitriptyline & N06AA09 \\
\hline Doxepin & Not approved in Japan \\
\hline Fluoxetine & Not approved in Japan \\
\hline Lithium & N05AN0I \\
\hline \multicolumn{2}{|l|}{ Antihistamines } \\
\hline Diphenhydramine & D04AA32 \\
\hline Doxylamine & Not approved in Japan \\
\hline \multicolumn{2}{|l|}{ Antimicrobials } \\
\hline Acyclivir & D06BB03 \\
\hline Aminoglycosides & JOIG \\
\hline Amphotericin B & $\mathrm{A} 0 \mathrm{IAB} 04$ \\
\hline Beta-lactam & JOIC, JOID \\
\hline Foscarnet & J05AD0I \\
\hline Ganciclovir & J05AB06 \\
\hline Pentamidine & POICXOI \\
\hline Quinolones & JOIM \\
\hline Rifanpin & J04AB02 \\
\hline Sulfonamides & JOIEEOI \\
\hline Vancomycin & A07AA09 \\
\hline \multicolumn{2}{|l|}{ Antiretrovials } \\
\hline Adefovir & J05AF08 \\
\hline Cidofovir & Not approved in Japan \\
\hline Tenofovir & J05AF07, J05AFI3, J05AR03, J05AR09, J05ARI7, J05ARI8 \\
\hline Indinavir & J05AE02 \\
\hline Benzodiazepines & N05BA, N05CD, N05CF \\
\hline \multicolumn{2}{|l|}{ Calcineurin inhibitors } \\
\hline Cyclosporine & L04AD0I \\
\hline Tacrolimus & L04AD02 \\
\hline \multicolumn{2}{|l|}{ Cardiovascular agents } \\
\hline Angiotensin-converting enzyme inhibitors & C09A, C09B \\
\hline Angiotensin receptor blockers & C09C, C09D \\
\hline Clopidogrel & $\mathrm{B} 0 \mathrm{IAC} 04$ \\
\hline Ticlopidine & B0IAC05 \\
\hline Statins & CIOAA, CIOBA \\
\hline \multicolumn{2}{|l|}{ Chemotherapeutics } \\
\hline Carmustine & LOIADOI \\
\hline Semustine & Not approved in Japan \\
\hline Cisplatin & LOIXAOI \\
\hline Interferon-alpha & L03ABOI, L03AB05, L03AB06, L03AB09, L03ABI0, L03ABII, L03ABI2, L03ABI5 \\
\hline Methotrexate & L04AX03 \\
\hline Mitomycin-C & L0IDC03 \\
\hline Contrast dye & V08A \\
\hline \multicolumn{2}{|l|}{ Diuretics } \\
\hline Loops & C03CA \\
\hline Thiazides & C03AA \\
\hline Triamterene & C03DB02 \\
\hline \multicolumn{2}{|l|}{ Proton pump inhibitors } \\
\hline Lansoprazole & $\mathrm{A02BC03}$ \\
\hline Omeprazole & $\mathrm{A} 02 \mathrm{BCOI}$ \\
\hline Pantoprazole & Not approved in Japan \\
\hline \multicolumn{2}{|l|}{ Others } \\
\hline Allopurinol & M04AA \\
\hline Gold therapy & $\mathrm{MOICB}$ \\
\hline Haloperidol & N05AD0I \\
\hline Pamidronate & М05ВА03 \\
\hline Phenytoin & N03AB02, N03AB52 \\
\hline Quinine & POIBCOI \\
\hline Zoledronate & M05BA08 \\
\hline
\end{tabular}

Abbreviation: ATC, Anatomical Therapeutic Chemical. 


\section{Publish your work in this journal}

Clinical Epidemiology is an international, peer-reviewed, open access, online journal focusing on disease and drug epidemiology, identification of risk factors and screening procedures to develop optimal preventative initiatives and programs. Specific topics include: diagnosis, prognosis, treatment, screening, prevention, risk factor modification,

Submit your manuscript here: https://www.dovepress.com/clinical-epidemiology-journal systematic reviews, risk and safety of medical interventions, epidemiology and biostatistical methods, and evaluation of guidelines, translational medicine, health policies and economic evaluations. The manuscript management system is completely online and includes a very quick and fair peer-review system, which is all easy to use. 